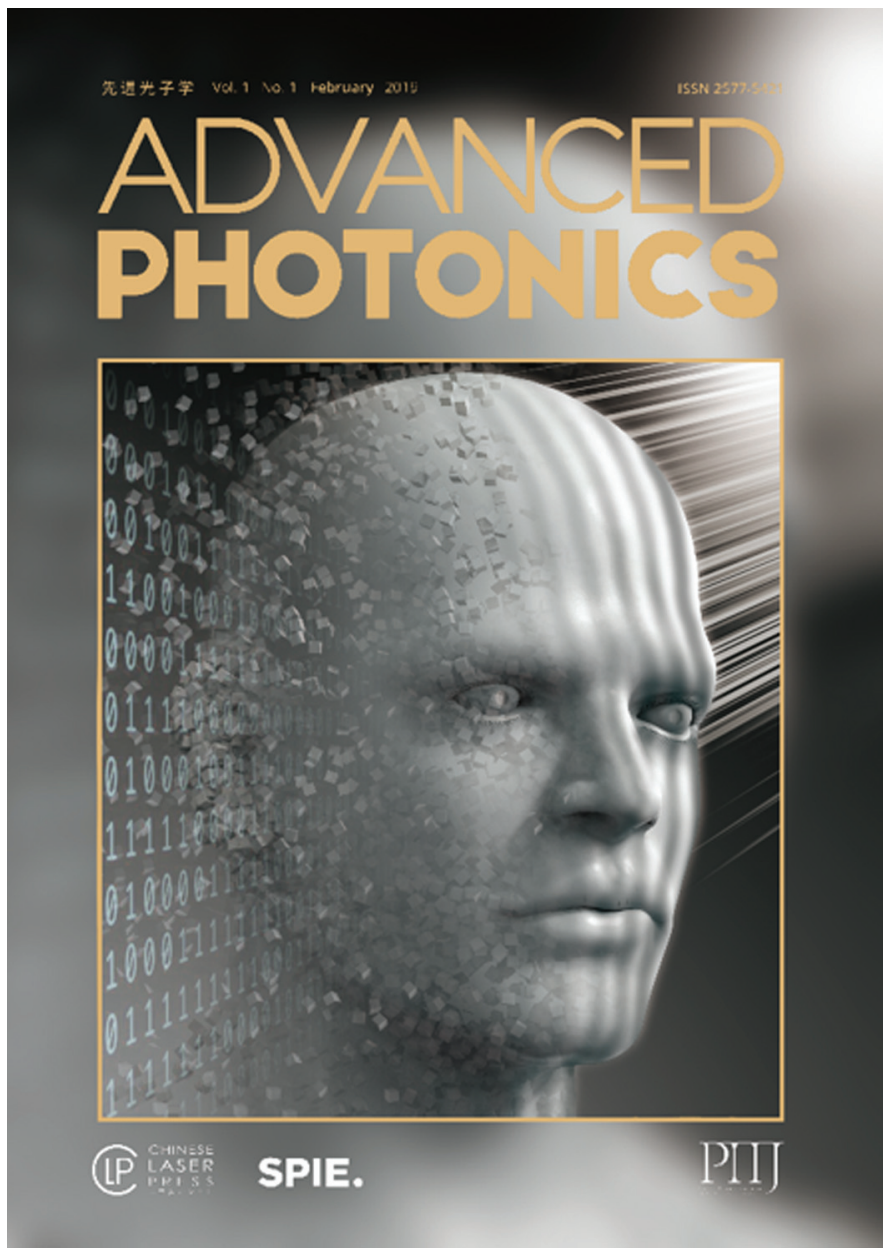

\title{
About the Cover: Advanced Photonics Volume 1, Issue 2
}

The image on the cover of Advanced Photonics Volume 1, Issue 2, exemplifies phase demodulation from a single fringe image utilizing deep learning and artificial intelligence. The 3D surface profile of the human face is encoded within the phase of the distorted sinusoidal fringe pattern, which is directly retrieved through deep neural networks with high accuracy and fidelity. Provided by S. Feng et al., a team of researchers from Nanjing University of Science and Technology, the image is based on the research presented in their article, "Fringe pattern analysis using deep learning" (DOI: 10/1117/1.AP.1.2.025001). 